\title{
Continuous Dependence of the Solutions of Nonlinear Integral Quadratic Volterra Equation on the Parameter
}

\author{
Szymon Dudek and Leszek Olszowy \\ Department of Mathematics, Rzeszów University of Technology, al. Powstańców Warszawy 6, 35-959 Rzeszów, Poland \\ Correspondence should be addressed to Szymon Dudek; sdudek@prz.edu.pl
}

Received 12 August 2014; Revised 13 October 2014; Accepted 13 October 2014

Academic Editor: Józef Banaś

Copyright (C) 2015 S. Dudek and L. Olszowy. This is an open access article distributed under the Creative Commons Attribution License, which permits unrestricted use, distribution, and reproduction in any medium, provided the original work is properly cited.

We prove results on the existence and continuous dependence of solutions of a nonlinear quadratic integral Volterra equation on a parameter. This dependence is investigated in terms of Hausdorff distance. The considerations are placed in the Banach space and the Fréchet space.

\section{Introduction}

In this paper we investigate the following nonlinear quadratic integral Volterra equation:

$$
x(t)=a(t)+f(t, x(t)) \int_{0}^{t} v(t, s, x(s)) d s, \quad t \in J,
$$

both on a bounded interval (i.e., $J=[0, T]$ ) and on an unbounded one $\left(J=\mathbb{R}_{+}=[0, \infty)\right)$, where $a, f$, and $v$ are given functions. We will study (1) in the Banach space $C([0, T])$ when $J=[0, T]$ and in the Fréchet space $C\left(\mathbb{R}_{+}\right)$ when $J=\mathbb{R}_{+}$.

The main aim of the paper is to formulate assumptions that guarantee continuous dependence of solutions of (1) on parameter. In our considerations we do not assume the uniqueness of solutions, while dependence of the set of solutions on a parameter will be expressed in terms of Hausdorff distance of the spaces $C([0, T])$ and $C\left(\mathbb{R}_{+}\right)$.

Quadratic integral equations appear in theories of radiative transfer and neutron transport and in kinetic theory of gases (cf. [1-4]). Up to this time, a lot of papers have appeared on those equations [1-9]; however, to the best of our knowledge, there are no papers on continuous dependence of solutions of this kind of equations on parameter.

Existence results for (1) have been obtained with the help of fixed point theorems expressed in terms of measures of nonconpactness.

\section{Notation and Auxiliary Facts}

In this section we collect some definitions and results which will be needed later. Assume that $E$ is a real Banach space with the norm $\|\cdot\|$ and the zero element $\theta$. Denote by $B(x, r)$ the closed ball centered at $x$ and with radius $r$. The ball $B(\theta, r)$ will be denoted by $B_{r}$. If $X$ is a subset of $E$, then the symbols $\bar{X}$ and $\operatorname{Conv} X$ stand for the closure and convex closure of $X$, respectively. The family of all nonempty and bounded subsets of $E$ will be denoted by $\mathfrak{M}_{E}$ while its subfamily consisting of all relatively compact sets is denoted by $\mathfrak{N}_{E}$. Following $[5,8,10]$ we accept the following definition of a measure of noncompactness.

Definition 1. A mapping $\mu: \mathfrak{M}_{E} \rightarrow \mathbb{R}_{+}$is said to be a measure of noncompactness if it satisfies the following conditions.

$\left(1^{o}\right)$ The family ker $\mu=\left\{X \in \mathfrak{M}_{E}: \mu(X)=0\right\}$ is nonempty and $\operatorname{ker} \mu \subset \mathfrak{N}_{E}$.

$\left(2^{\circ}\right) X \subset Y \Rightarrow \mu(X) \leq \mu(Y)$.

$\left(3^{o}\right) \mu(\bar{X})=\mu(\operatorname{Conv} X)=\mu(X)$.

$\left(4^{o}\right) \mu(\lambda X+(1-\lambda) Y) \leq \lambda \mu(X)+(1-\lambda) \mu(Y)$ for $\lambda \in[0,1]$.

$\left(5^{\circ}\right)$ If $\left(X_{k}\right)_{k \in \mathbb{N}}$ is a sequence of closed sets from $\mathfrak{M}_{E}$ such that $X_{k+1} \subset X_{k}(k=1,2, \ldots)$ and if $\lim _{k \rightarrow \infty} \mu\left(X_{k}\right)=$ 0 , then the intersection set $X_{\infty}=\bigcap_{k=1}^{\infty} X_{k}$ is nonempty. 
For our purposes we will only need the following fixed point theorem $[6,8-11]$.

Theorem 2. Let $\Omega$ be nonempty bounded closed convex subset of the space $E$ and let $V: \Omega \rightarrow \Omega$ be continuous such that $\mu(V X) \leq k \mu(X)$ for any nonempty subset $X$ of $\Omega$, where $k$ is a constant, $k \in[0,1)$. Then $V$ has a fixed point in the set $\Omega$.

In the sequel we will work in the Banach space $C([0, T])$ consisting of all real functions defined and continuous on $[0, T]$. The space $C([0, T])$ is furnished with the standard norm

$$
\|x\|=\sup \{|x(t)|: t \in[0, T]\} .
$$

Now we recollect the definition of the measure of noncompactness which will be used further on. This measure was introduced in $[8,10]$. Fix a nonempty bounded subset $X$ of $C([0, T])$ and a positive number $T>0$. For $x \in X$ and $\varepsilon>0$ let us denote by $\omega(x, \varepsilon)$ the modulus of continuity of the function $x$ on the interval $[0, T]$; that is,

$$
\omega^{T}(x, \varepsilon)=\sup \{|x(t)-x(s)|: t, s \in[0, T],|t-s| \leq \varepsilon\} .
$$

Further, let us put

$$
\begin{gathered}
\omega^{T}(X, \varepsilon)=\sup \left\{\omega^{T}(x, \varepsilon): x \in X\right\}, \\
\omega_{0}^{T}(X)=\lim _{\varepsilon \rightarrow 0+} \omega^{T}(X, \varepsilon) .
\end{gathered}
$$

It can be shown $[9,10]$ that the function $\omega_{0}^{T}$ is a measure of noncompactness in the space $C([0, T])$.

In what follows, we will also work in the space $C\left(\mathbb{R}_{+}\right)$ consisting of all real functions defined and continuous on $\mathbb{R}_{+}$. The space $C\left(\mathbb{R}_{+}\right)$equipped with the family of seminorms

$$
\|x\|_{n}=\sup \{|x(t)|: t \in[0, n]\}, \quad n \in \mathbb{N},
$$

becomes a Fréchet space furnished with the distance

$$
\begin{array}{r}
d_{C}(x, y)=\sup \left\{2^{-n} \frac{\|x-y\|_{n}}{1+\|x-y\|_{n}}: n \in \mathbb{N}\right\}, \\
x, y \in C\left(\mathbb{R}_{+}\right),
\end{array}
$$

or equivalently

$$
\widetilde{d}_{C}(x, y)=\sum_{n=1}^{\infty} 2^{-n} \frac{\|x-y\|_{n}}{1+\|x-y\|_{n}}, \quad x, y \in C\left(\mathbb{R}_{+}\right) .
$$

A nonempty subset $X \subset C\left(\mathbb{R}_{+}\right)$is said to be bounded if

$$
\sup \left\{\|x\|_{n}: x \in X\right\}<\infty
$$

for $n \in \mathbb{N}$.
Further, let $\mathfrak{M}_{C}$ denote the family of all nonempty and bounded subsets of $C\left(\mathbb{R}_{+}\right)$and $\mathfrak{N}_{C}$ the family of all relatively compact subsets of $C\left(\mathbb{R}_{+}\right)$. Obviously $\mathfrak{N}_{C} \subset \mathfrak{M}_{C}$.

We accept the following definition of the notion of a sequence of measures of noncompactness $[12,13]$.

Definition 3. A sequence of functions $\left\{\mu_{n}\right\}_{n \in \mathbb{N}}$, where $\mu_{n}$ : $\mathfrak{M}_{C} \rightarrow[0, \infty)$, is said to be a sequence of measures of noncompactness in $C\left(\mathbb{R}_{+}\right)$if it satisfies the following conditions.

$\left(1^{o}\right)$ The family $\operatorname{ker}\left\{\mu_{n}\right\}=\left\{X \in \mathfrak{M}_{C}: \mu_{n}(X)=0\right.$ for $n \in$ $\mathbb{N}\}$ is nonempty and $\operatorname{ker}\left\{\mu_{n}\right\} \subset \mathfrak{N}_{C}$.

$\left(2^{o}\right) X \subset Y \Rightarrow \mu_{n}(X) \leq \mu_{n}(Y)$ for $n \in \mathbb{N}$.

$\left(3^{o}\right) \mu_{n}(\bar{X})=\mu_{n}(\operatorname{Conv} X)=\mu_{n}(X)$ for $n \in \mathbb{N}$.

$\left(4^{o}\right)$ If $\left(X_{k}\right)_{k \in \mathbb{N}}$ is a sequence of closed sets from $\mathfrak{M}_{C}$ such that $X_{k+1} \subset X_{k}(k=1,2, \ldots)$ and if $\lim _{k \rightarrow \infty} \mu_{n}\left(X_{k}\right)=0$ for each $n \in \mathbb{N}$, then the intersection set $X_{\infty}=\bigcap_{k=1}^{\infty} X_{k}$ is nonempty.

We have the following two facts (see $[12,13])$.

Theorem 4. The family of mappings $\left\{\omega_{0}^{n}\right\}_{n \in \mathbb{N}}$, where $\omega_{0}^{n}$ : $\mathfrak{M}_{C} \rightarrow[0, \infty)$, satisfies the conditions $\left(1^{o}\right)-\left(4^{o}\right)$ from Definition 3 and, moreover, $\operatorname{ker}\left\{\omega_{n}\right\}=\mathfrak{N}_{C}$.

Theorem 5. Let $\Omega$ be a nonempty, bounded, closed, and convex subset of the space $C\left(\mathbb{R}_{+}\right)$and let $V: \Omega \rightarrow \Omega$ be continuous mapping. Suppose that there exists a sequence of numbers $\left(k_{n}\right) \subset[0,1)$ such that

$$
\omega_{0}^{n}(V X) \leq k_{n} \omega_{0}^{n}(X)
$$

for nonempty $X \subset \Omega$ and $n \in \mathbb{N}$. Then $V$ has at least one fixed point in the set $\Omega$.

Let $(Z, d)$ be an arbitrary metric space. For any two nonempty and bounded subsets of $Z$ we define their Hausdorff distance $D_{Z}(X, Y)$ by formula

$$
D_{Z}(X, Y):=\inf \{r \geq 0: X \subset B(Y, r), Y \subset B(X, r)\}
$$

where

$$
B(X, r):=\bigcup_{x \in X}\{z \in Z: d(x, z) \leq r\} .
$$

In the next chapters, we will consider Hausdorff distance $D_{C([0, T])}$ in the family $\mathfrak{N}_{C([0, T])}$ of nonempty and relatively compact subsets of the Banach space $C[0, T]$ and $D_{C\left(\mathbb{R}_{+}\right)}$in the family $\mathfrak{N}_{C\left(\mathbb{R}_{+}\right)}$of nonempty and relatively compact subsets of the Fréchet space $C\left(\mathbb{R}_{+}\right)$(with distance $d_{C}$ ).

Let $(\Lambda, d)$ be arbitrary metric space, and let us consider a mapping $F: \Lambda \rightarrow \mathfrak{N}_{C\left(\mathbb{R}_{+}\right)}$. Since we will consider the continuity of such mappings (with respect to the distance $D_{C\left(\mathbb{R}_{+}\right)}$ in $\mathfrak{N}_{C\left(\mathbb{R}_{+}\right)}$), we need the following lemma. 
Lemma 6. A mapping $F: \Lambda \rightarrow \mathfrak{N}_{C\left(\mathbb{R}_{+}\right)}$is continuous at a point $\lambda_{0} \in \Lambda$ if and only if

$$
\begin{aligned}
& \forall_{\varepsilon>0} \forall_{n \in \mathbb{N}} \exists_{\delta>0} \forall_{\lambda \in \Lambda, d\left(\lambda_{0}, \lambda\right)<\delta} \forall_{x \in F\left(\lambda_{0}\right)} \exists_{y \in F(\lambda)}\|x-y\|_{n} \leq \varepsilon, \\
& \forall_{\varepsilon>0} \forall_{n \in \mathbb{N}} \exists_{\delta>0} \forall_{\lambda \in \Lambda, d\left(\lambda_{0}, \lambda\right)<\delta} \forall_{y \in F(\lambda)} \exists_{x \in F\left(\lambda_{0}\right)}\|x-y\|_{n} \leq \varepsilon .
\end{aligned}
$$

Proof. From (6) we derive that for $\eta>0$ we have

$$
\begin{aligned}
d_{C}(x, y) \leq \eta \Longleftrightarrow & \|x-y\|_{k} \leq \frac{\eta}{2^{-k}-\eta}, \\
& \text { for } k=1, \ldots, n(\eta),
\end{aligned}
$$

where

$$
n(\eta):= \begin{cases}\max \left\{k \in \mathbb{N}: k<\log _{2} \frac{1}{\eta}\right\}, & \text { for } \eta \leq \frac{1}{2}, \\ 0, & \text { for } \eta>\frac{1}{2} .\end{cases}
$$

The continuity of the mapping $F \rightarrow \mathfrak{N}_{C\left(\mathbb{R}_{+}\right)}$at a point $\lambda_{0} \in \Lambda$ means

$$
\begin{aligned}
& \forall_{\varepsilon>0} \exists_{\delta>0} \forall_{\lambda \in \Lambda, d\left(\lambda_{0}, \lambda\right)<\delta} \forall_{x \in F\left(\lambda_{0}\right)} \exists_{y \in F(\lambda)} d_{C}(x, y) \leq \varepsilon, \\
& \forall_{\varepsilon>0} \exists_{\delta>0} \forall_{\lambda \in \Lambda, d\left(\lambda_{0}, \lambda\right)<\delta} \forall_{y \in F(\lambda)} \exists_{x \in F\left(\lambda_{0}\right)} d_{C}(x, y) \leq \varepsilon .
\end{aligned}
$$

We will show that conditions (12) and (13) are equivalent to conditions (16) and (17).

First we suppose that (12) and (13) are fulfilled. Let us fix $\varepsilon>0$. The condition (12) guarantees that there exist numbers $\delta_{k}>0, k=1, \ldots, n(\varepsilon)$, such that

$$
\forall_{\lambda \in \Lambda, d\left(\lambda_{0}, \lambda\right)<\delta_{k}} \forall_{x \in F\left(\lambda_{0}\right)} \exists_{y \in F(\lambda)}\|x-y\|_{k} \leq \frac{\varepsilon}{2^{-k}-\varepsilon}
$$

for $k=1, \ldots, n(\varepsilon)$.

Let us put $\delta:=\min \left\{\delta_{k}: k=1, \ldots, n(\varepsilon)\right\}$. Applying (14) we get

$$
\forall_{\lambda \in \Lambda, d\left(\lambda_{0}, \lambda\right)<\delta} \forall_{x \in F\left(\lambda_{0}\right)} \exists_{y \in F(\lambda)} d_{C}(x, y) \leq \varepsilon .
$$

In a similar way we show that (17) is also satisfied.

Now we assume that (16) and (17) are fulfilled. We will prove conditions (12) and (13). Let us fix $\varepsilon>0$ and $n \in$ $\mathbb{N}$. There exists a number $\eta>0$ such that $\eta<2^{-n}$ and $\eta /\left(2^{-n}-\eta\right)=\varepsilon$. Condition (16) implies that there is a number $\delta>0$ such that

$$
\forall_{\lambda \in \Lambda, d\left(\lambda_{0}, \lambda\right)<\delta} \forall_{x \in F\left(\lambda_{0}\right)} \exists_{y \in F(\lambda)} d_{C}(x, y) \leq \eta .
$$

Hence, using (14) we obtain that

$$
\|x-y\|_{n} \leq \frac{\eta}{2^{-n}-\eta}=\varepsilon
$$

and therefore (16) is confirmed. In a similar way we show that condition (17) is also satisfied.

\section{Existence Result}

In this section we give an existence result for the following nonlinear integral Volterra equation:

$$
x(t)=a(t)+f(t, x(t)) \int_{0}^{t} v(t, s, x(s)) d s, \quad t \in[0, T] .
$$

In the last years there have been published a few dozen papers on nonlinear quadratic integral equations. From among papers cited here, majority of them was concerned with different kinds of equations from (22); see $[5-7,13]$. The authors of papers $[8,9,11,13]$ examined equations similar to (22); however, their considerations were conducted in the Banach space $B C\left(\mathbb{R}_{+}\right)$and therefore their assumptions were too restrictive. In the paper [12] we investigated similar equations to (22) with different kinds of assumptions than these given below.

This theorem will be a starting point of our further investigations on the continuous dependence of solutions on parameter.

Observe that the above equation includes several classes of functional, integral, and functional integral equations considered in the literature [1-4].

Equation (22) will be considered under the following assumptions.

$\left(H_{1}\right)$ The function $a:[0, T] \rightarrow \mathbb{R}$ is continuous on $[0, T]$.

$\left(H_{2}\right)$ The function $f:[0, T] \times \mathbb{R} \rightarrow \mathbb{R}$ is continuous and there exists a constant $k_{f}$ such that $|f(t, x)-f(t, y)| \leq$ $k_{f}|x-y|$ for any $t \in[0, T]$ and for all $x, y \in \mathbb{R}$.

$\left(H_{3}\right)$ The function $v:[0, T] \times[0, T] \times \mathbb{R} \rightarrow \mathbb{R}$ is continuous. Moreover, there exists a function $g$ : $[0, T] \times[0, T] \rightarrow \mathbb{R}_{+}$being continuous on $[0, T] \times$ $[0, T]$ such that

$$
|v(t, s, x)| \leq g(t, s)
$$

for all $t, s \in[0, T], x \in \mathbb{R}$.

$\left(H_{4}\right)$ Consider

$$
k_{f} \sup _{t \in[0, T]} \int_{0}^{t} g(t, s) d s<1
$$

Then we can formulate our existence result.

Theorem 7. Under assumptions $\left(H_{1}\right)-\left(H_{4}\right),(22)$ has at least one solution in the space $C([0, T])$.

Proof. Consider the operator $V: C([0, T]) \rightarrow C([0, T])$ defined by the formula

$$
(V x)(t):=a(t)+f(t, x(t)) \int_{0}^{t} v(t, s, x(s)) d s, \quad t \in[0, T] .
$$


Using assumptions $\left(H_{1}\right)-\left(H_{3}\right)$ and reasoning similarly as in [5-11], we can show that the operator $V$ is well defined and is continuous on $C([0, T])$. Next let us put

$$
\begin{aligned}
r:= & \left(1-k_{f} \sup _{t \in[0, T]} \int_{0}^{t} g(t, s) d s\right)^{-1} \\
& \times\left(\sup _{t \in[0, T]}|a(t)|+\sup _{t \in[0, T]}|f(t, 0)| \sup _{t \in[0, T]} \int_{0}^{t} g(t, s) d s\right) .
\end{aligned}
$$

Notice that for $x \in B(\theta, r)$ we have in virtue of $\left(H_{1}\right)-\left(H_{3}\right)$

$$
\begin{aligned}
|(V x)(t)| \leq & \sup _{t \in[0, T]}|a(t)| \\
& +\left(\sup _{t \in[0, T]}|f(t, 0)|+k_{f} r\right) \sup _{t \in[0, T]} \int_{0}^{t} g(t, s) d s=r
\end{aligned}
$$

for $t \in[0, T]$ and therefore the operator $V$ transforms the ball $B(\theta, r)$ into itself.

Now, let us take a nonempty subset $X$ of the ball $B(\theta, r)$ and fix $x \in X$. Choose $t_{1}, t_{2} \in[0, T]$ such that $\left|t_{2}-t_{1}\right|<\varepsilon$. Without loss of generality we can assume that $t_{2}>t_{1}$. Then we obtain

$$
\begin{aligned}
& \left|(V x)\left(t_{2}\right)-(V x)\left(t_{1}\right)\right| \\
& \leq\left|a\left(t_{2}\right)-a\left(t_{1}\right)\right| \\
& +\left(\left|f\left(t_{2}, x\left(t_{2}\right)\right)-f\left(t_{1}, x\left(t_{2}\right)\right)\right|\right. \\
& \left.\quad+\left|f\left(t_{1}, x\left(t_{2}\right)\right)-f\left(t_{1}, x\left(t_{1}\right)\right)\right|\right) \\
& \quad \times \int_{0}^{t_{2}}\left|v\left(t_{2}, s, x(s)\right)\right| d s+\left|f\left(t_{1}, x\left(t_{1}\right)\right)\right| \\
& \quad \times\left(\int_{t_{1}}^{t_{2}}\left|v\left(t_{2}, s, x(s)\right)\right| d s\right. \\
& \left.\quad+\int_{0}^{t_{1}}\left|v\left(t_{2}, s, x(s)\right)-v\left(t_{1}, s, x(s)\right)\right| d s\right) \\
& \leq \xi^{T}(a, \varepsilon)+\left(\xi^{T}(f, \varepsilon)+k_{f} \omega^{T}(x, \varepsilon)\right) \sup _{t \in[0, T]} \int_{0}^{t} g(t, s) d s \\
& +\bar{F}\left(\varepsilon \bar{V}+T \xi^{T}(v, \varepsilon)\right),
\end{aligned}
$$

where we denoted

$$
\begin{gathered}
\xi^{T}(a, \varepsilon):=\sup \left\{\left|a\left(t_{2}\right)-a\left(t_{1}\right)\right|: t_{2}, t_{1}, \in[0, T],\left|t_{2}-t_{1}\right|<\varepsilon\right\}, \\
\xi^{T}(f, \varepsilon):=\sup \left\{\left|f\left(t_{2}, x\right)-f\left(t_{1}, x\right)\right|: t_{2}, t_{1}, \in[0, T],\right. \\
\left.\left|t_{2}-t_{1}\right|<\varepsilon,|x| \leq r\right\},
\end{gathered}
$$

$$
\begin{aligned}
\xi^{T}(v, \varepsilon):=\sup \left\{\left|v\left(t_{2}, s, x\right)-v\left(t_{1}, s, x\right)\right|: t_{2}, t_{1}, s \in[0, T],\right. \\
\left.\left|t_{2}-t_{1}\right|<\varepsilon,|x| \leq r\right\}, \\
\bar{F}:=\sup \{|f(t, x)|: t \in[0, T],|x| \leq r\}, \\
\bar{V}:=\sup \{|v(t, s, x)|: t, s \in[0, T],|x| \leq r\} .
\end{aligned}
$$

From the above estimate we derive the following one:

$$
\begin{aligned}
\omega^{T}(V X, \varepsilon) \leq & \xi^{T}(a, \varepsilon)+\xi^{T}(f, \varepsilon) \sup _{t \in[0, T]} \int_{0}^{t} g(t, s) d s \\
& +k_{f} \sup _{t \in[0, T]} \int_{0}^{t} g(t, s) d s \cdot \omega^{T}(X, \varepsilon) \\
& +\bar{F}\left(\varepsilon \bar{V}+T \xi^{T}(v, \varepsilon)\right) .
\end{aligned}
$$

Observe that $\xi^{T}(a, \varepsilon) \rightarrow 0, \xi^{T}(f, \varepsilon) \rightarrow 0$, and $\xi^{T}(v, \varepsilon) \rightarrow 0$ as $\varepsilon \rightarrow 0$, which are simple consequences of the uniform continuity of the functions $a, f$, and $v$ on the sets $[0, T]$, $\{(t, x): t \in[0, T],|x| \leq r\}$, and $\{(t, s, x): t, s \in[0, T],|x| \leq r\}$, respectively. Hence we get

$$
\omega_{0}^{T}(V X) \leq k_{f} \sup _{t \in[0, T]} \int_{0}^{t} g(t, s) d s \cdot \omega_{0}^{T}(X) .
$$

Since $k_{f} \sup _{t \in[0, T]} \int_{0}^{t} g(t, s) d s<1$ in view of assumption $\left(H_{4}\right)$, from the above estimate and Theorem 2 we deduce that the operator $V$ has a fixed point $x$ in the set $B(\theta, r)$. Obviously $x$ is a solution of the functional integral equation (22).

Our next result is concerned with (22) on the real halfaxis $\mathbb{R}_{+}$. Let us consider equation

$$
x(t)=a(t)+f(t, x(t)) \int_{0}^{t} v(t, s, x(s)) d s, \quad t \in \mathbb{R}_{+} .
$$

Equation (32) will be considered under the following assumptions.

$\left(H_{1}^{\prime}\right)$ The function $a: \mathbb{R}_{+} \rightarrow \mathbb{R}$ is continuous on $\mathbb{R}_{+}$.

$\left(H_{2}^{\prime}\right)$ The function $f: \mathbb{R}_{+} \times \mathbb{R} \rightarrow \mathbb{R}$ is continuous and there exists a constant $k_{f}$ such that $|f(t, x)-f(t, y)| \leq$ $k_{f}|x-y|$ for any $t \in \mathbb{R}_{+}$and for all $x, y \in \mathbb{R}$.

$\left(H_{3}^{\prime}\right)$ The function $v: \mathbb{R}_{+} \times \mathbb{R}_{+} \times \mathbb{R} \rightarrow \mathbb{R}$ is continuous. Moreover, there exists a function $g: \mathbb{R}_{+} \times \mathbb{R}_{+} \rightarrow \mathbb{R}_{+}$ being continuous on $\mathbb{R}_{+} \times \mathbb{R}_{+}$such that

$$
|v(t, s, x)| \leq g(t, s)
$$

for all $t, s \in \mathbb{R}_{+}, x \in \mathbb{R}$.

$\left(H_{4}^{\prime}\right)$ Consider

$$
k_{f} \sup _{t \in[0, T]} \int_{0}^{t} g(t, s) d s<1, \quad T \in \mathbb{R}_{+} .
$$

Then we can formulate the next existence result. 
Theorem 8. Under assumptions $\left(H_{1}^{\prime}\right)-\left(H_{4}^{\prime}\right)$, (32) has at least one solution in the space $C\left(\mathbb{R}_{+}\right)$.

Proof. In contrast to papers $[5,6,8,9,11]$, we place our considerations in the Fréchet space $C\left(\mathbb{R}_{+}\right)$, instead of the Banach space $B C\left(\mathbb{R}_{+}\right)$.

Let us define the operator $V: C\left(\mathbb{R}_{+}\right) \rightarrow C\left(\mathbb{R}_{+}\right)$defined by the formula

$$
(V x)(t):=a(t)+f(t, x(t)) \int_{0}^{t} v(t, s, x(s)) d s, \quad t \in \mathbb{R}_{+} .
$$

Using assumptions $\left(H_{1}^{\prime}\right)-\left(H_{3}^{\prime}\right)$ and standard arguments, we can show that the operator $V$ is well defined and is continuous on $C\left(\mathbb{R}_{+}\right)$. Next let us put

$$
\begin{aligned}
r(t):= & \left(1-k_{f} \int_{0}^{t} g(t, s) d s\right)^{-1} \\
& \times\left(|a(t)|+|f(t, 0)| \int_{0}^{t} g(t, s) d s\right), \quad t \in \mathbb{R}_{+}, \\
\Omega & :=\left\{x \in C\left(\mathbb{R}_{+}\right):|x(t)| \leq r(t) \text { for } t \in \mathbb{R}_{+}\right\} .
\end{aligned}
$$

Notice that for $x \in \Omega$ we have in virtue of $\left(H_{1}^{\prime}\right)-\left(H_{3}^{\prime}\right)$ and (36)

$$
|(V x)(t)| \leq|a(t)|+\left(|f(t, 0)|+k_{f} r(t)\right) \int_{0}^{t} g(t, s) d s=r(t)
$$

and therefore the operator $V$ transforms $\Omega$ into itself.

Applying (31) with $T=n, n \in \mathbb{N}$, we get for $X \subset \Omega$

$$
\omega_{0}^{n}(V X) \leq k_{f} \sup _{t \in[0, n]} \int_{0}^{t} g(t, s) d s \cdot \omega_{0}^{n}(X) .
$$

Since $k_{f} \sup _{t \in[0, n]} \int_{0}^{t} g(t, s) d s<1$ in view of assumption $\left(H_{4}^{\prime}\right)$, from the above estimate and Theorem 5 , we deduce that the operator $V$ has a fixed point $x$ in the set $\Omega$. Obviously $x$ is a solution of the functional integral equation (22).

\section{Continuous Dependence of Solutions on Parameter and the Examples}

In this section we will investigate (1) depending on parameter $\lambda$; that is, we will consider equation of the type

$$
x(t)=a(\lambda, t)+f(\lambda, t, x(t)) \int_{0}^{t} v(\lambda, t, s, x(s)) d s, \quad t \in J,
$$

where $\lambda$ is an element of metric space $(\Lambda, d), J=[0, T]$ or $J=\mathbb{R}_{+}$.

For fixed $\lambda \in \Lambda$ we will denote the operator $V_{\lambda}: C(J) \rightarrow$ $C(J)$ specified by formula

$$
\begin{array}{r}
\left(V_{\lambda} x\right)(t):=a(\lambda, t)+f(\lambda, t, x(t)) \int_{0}^{t} v(\lambda, t, s, x(s)) d s, \\
x \in C(J), \quad t \in J,
\end{array}
$$

and put

$$
\operatorname{Fix}(\lambda):=\left\{x \in C(J): V_{\lambda} x=x\right\} .
$$

Obviously $\operatorname{Fix}(\lambda)$ is the set of all solutions of (40) and it is compact in view of condition (31), so $\operatorname{Fix}(\lambda) \in \mathfrak{N}_{C(J)}$.

The aim of this paper is to provide the conditions concerning the functions involved in (40), which imply that the sets $\operatorname{Fix}(\lambda)$ are nonempty and change continuously in the space $C(J)$ with respect to parameter $\lambda$ (in view of Hausdorff metric $\left.D_{C(J)}\right)$.

First we consider case of the bounded set $J=[0, T]$. Let us take the following assumptions.

$\left(A_{1}\right) a: \Lambda \times[0, T] \rightarrow \mathbb{R}$ is a function such that the function $t \mapsto a(\lambda, t)$ is continuous on $[0, T]$ for arbitrarily fixed $\lambda \in \Lambda$ and moreover there exists a nondecreasing function $p_{a}: \mathbb{R}_{+} \rightarrow \mathbb{R}_{+}$, continuous at 0 and $p_{a}(0)=$ 0 , such that

$$
\left|a\left(\lambda_{1}, t\right)-a\left(\lambda_{2}, t\right)\right| \leq p_{a}\left(d\left(\lambda_{1}, \lambda_{2}\right)\right)
$$

for $\lambda_{1}, \lambda_{2} \in \Lambda, t \in[0, T]$.

$\left(A_{2}\right) f: \Lambda \times[0, T] \times \mathbb{R} \rightarrow \mathbb{R}$ is a function such that the function $(t, x) \mapsto f(\lambda, t, x)$ is continuous and bounded on the set $[0, T] \times \mathbb{R}$ for arbitrarily fixed $\lambda \in \Lambda$ and there are a constant $k_{f}$ and a nondecreasing function $p_{f}: \mathbb{R}_{+} \rightarrow \mathbb{R}_{+}$, continuous at 0 and $p_{f}(0)=$ 0 , such that

$$
|f(\lambda, t, x)-f(\lambda, t, y)| \leq k_{f}|x-y|
$$

for any $\lambda \in \Lambda, t \in[0, T], x, y \in \mathbb{R}$, and

$$
\left|f\left(\lambda_{1}, t, x\right)-f\left(\lambda_{2}, t, x\right)\right| \leq p_{f}\left(d\left(\lambda_{1}, \lambda_{2}\right)\right)
$$

for all $\lambda_{1}, \lambda_{2} \in \Lambda, t \in[0, T], x \in \mathbb{R}$.

$\left(A_{3}\right) v: \Lambda \times[0, T] \times[0, T] \times \mathbb{R} \rightarrow \mathbb{R}$ is a function such that the function $(t, s, x) \mapsto v(\lambda, t, s, x)$ is continuous on the set $[0, T] \times[0, T] \times \mathbb{R}$ for arbitrarily fixed $\lambda \in \Lambda$ and there are a continuous function $g:[0, T] \times[0, T] \rightarrow$ $\mathbb{R}_{+}$and a nondecreasing function $p_{v}: \mathbb{R}_{+} \rightarrow \mathbb{R}_{+}$, continuous at 0 and $p_{v}(0)=0$, such that

$$
|v(\lambda, t, s, x)| \leq g(t, s)
$$

for all $\lambda \in \Lambda, t, s \in[0, T], x \in \mathbb{R}$, and

$$
\left|v\left(\lambda_{1}, t, s, x\right)-v\left(\lambda_{2}, t, s, x\right)\right| \leq p_{v}\left(d\left(\lambda_{1}, \lambda_{2}\right)\right)
$$

for all $\lambda_{1}, \lambda_{2} \in \Lambda, t, s \in[0, T], x \in \mathbb{R}$.

$\left(A_{4}\right)$ Consider

$$
k_{f} \sup _{t \in[0, T]} \int_{0}^{t} g(t, s) d s<1 .
$$

The following example shows that conditions $\left(A_{1}\right)-\left(A_{4}\right)$ are not enough for continuous dependence of the solutions on parameter $\lambda$. 
Example 9. Let us consider the equation

$$
x(t)=\int_{0}^{t} \sqrt{\lambda+\min \{|x(s)|, 2+2 \sqrt{2}\}} d s, \quad t \in[0,2],
$$

where $\lambda \in \Lambda:=[0,1]$. The space $\Lambda$ is equipped with the standard Euclidean metric. Obviously it is a particular case of (40), where

$$
\begin{gathered}
a(\lambda, t) \equiv 0, \quad f(\lambda, t, x) \equiv 1, \quad k_{f} \equiv 0, \\
v(\lambda, t, s, x)=\sqrt{\lambda+\min \{|x|, 2+2 \sqrt{2}\}}, \\
g(t, s)=\sqrt{3+2 \sqrt{2}}, \quad p_{a}(\tau)=p_{f}(\tau)=\tau, \\
p_{v}(\tau)=\sqrt{\tau} .
\end{gathered}
$$

Notice that if $x$ is a solution of (49), then

$$
|x(t)| \leq \int_{0}^{2} \sqrt{1+2+2 \sqrt{2}} d s \leq 2 \sqrt{3+2 \sqrt{2}}=2+2 \sqrt{2}
$$

which shows that (49) may be represented by simpler form

$$
x(t)=\int_{0}^{t} \sqrt{\lambda+|x(s)|} d s, \quad t \in[0,2] .
$$

Using standard calculations we obtain that $\operatorname{Fix}(0)=\left\{x_{1}, x_{2}\right\}$, where $x_{1}(t) \equiv 0$ and $x_{2}(t)=t^{2} / 4$, but for $\lambda>0$ we have $\operatorname{Fix}(\lambda)=\{x\}$, where $x(t)=t(t / 4+\sqrt{\lambda})$. It is easy to check whether the sets $\operatorname{Fix}(\lambda)$ do not change continuously in point $\lambda=0$ (in view of Hausdorff metric). It means the conditions $\left(A_{1}\right)-\left(A_{4}\right)$, which guarantee the existence of the solutions together with Theorem 7 , are not sufficient for continuous dependence of the solutions on the parameter $\lambda$. Thus, we decide to take an additional assumption.

$\left(A_{5}\right)$ There is an integrable function $k_{v}:[0, T] \rightarrow \mathbb{R}_{+}$such that

$$
|v(\lambda, t, s, x)-v(\lambda, t, s, y)| \leq k_{v}(s)|x-y|
$$

for $\lambda \in \Lambda, t, s \in[0, T], x, y \in \mathbb{R}$.

Theorem 10. Under assumptions $\left(A_{1}\right)-\left(A_{5}\right),(40)$ has at least one solution in the space $C([0, T])$ for all parameters $\lambda \in \Lambda$ and moreover the set of all solutions $\operatorname{Fix}(\lambda)$ depends continuously (with respect to Hausdorff metric $D_{C([0, T])}$ ) on the parameter $\lambda$.

Proof. Theorem 7 implies that (40) has at least one solution for all $\lambda$.

Let us fix arbitrarily $\lambda \in \Lambda$ and $\varepsilon>0$. Since we want to prove that Fix : $\Lambda \rightarrow \mathfrak{N}_{C([0, T])}$ is continuous in point $\lambda$ with respect to Hausdorff metric $D_{C([0, T])}$, we should show that there exists $\delta>0$ such that

$$
\begin{aligned}
& \forall_{x \in \operatorname{Fix}(\lambda)} \forall_{\lambda_{1} \in B(\lambda, \delta)} \exists_{x_{1} \in \operatorname{Fix}\left(\lambda_{1}\right)}\left\|x-x_{1}\right\|<\varepsilon, \\
& \forall_{\lambda_{1} \in B(\lambda, \delta)} \forall_{x_{1} \in \operatorname{Fix}\left(\lambda_{1}\right)} \exists_{x \in \operatorname{Fix}(\lambda)}\left\|x-x_{1}\right\|<\varepsilon .
\end{aligned}
$$

Let us fix $x \in \operatorname{Fix}(\lambda)$ and choose arbitrarily $\lambda_{1} \in \Lambda, d\left(\lambda, \lambda_{1}\right)<$ 1. Then, for any $y \in C([0, T])$ we obtain the following estimation:

$$
\begin{aligned}
& \left|x(t)-\left(V_{\lambda_{1}} y\right)(t)\right| \\
& =\left|\left(V_{\lambda} x\right)(t)-\left(V_{\lambda_{1}} y\right)(t)\right| \leq\left|\left(V_{\lambda} x\right)(t)-\left(V_{\lambda} y\right)(t)\right| \\
& +\left|\left(V_{\lambda} y\right)(t)-\left(V_{\lambda_{1}} y\right)(t)\right| \\
& \leq \mid f(\lambda, t, x(t)) \int_{0}^{t} v(\lambda, t, s, x(s)) d s \\
& -f(\lambda, t, y(t)) \int_{0}^{t} v(\lambda, t, s, y(s)) d s \mid \\
& +\left|a(\lambda, t)-a\left(\lambda_{1}, t\right)\right| \\
& +\mid f(\lambda, t, y(t)) \int_{0}^{t} v(\lambda, t, s, y(s)) d s \\
& -f\left(\lambda_{1}, t, y(t)\right) \int_{0}^{t} v\left(\lambda_{1}, t, s, y(s)\right) d s \\
& \leq|f(\lambda, t, x(t))-f(\lambda, t, y(t))| \int_{0}^{t} v(\lambda, t, s, x(s)) d s \\
& +|f(\lambda, t, y(t))| \int_{0}^{t}|v(\lambda, t, s, x(s))-v(\lambda, t, s, y(s))| d s \\
& +p_{a}\left(d\left(\lambda, \lambda_{1}\right)\right)+\left|f(\lambda, t, y(t))-f\left(\lambda_{1}, t, y(t)\right)\right| \\
& \times \int_{0}^{t}|v(\lambda, t, s, y(s))| d s+\left|f\left(\lambda_{1}, t, y(t)\right)\right| \\
& \times \int_{0}^{t}\left|v(\lambda, t, s, y(s))-v\left(\lambda_{1}, t, s, y(s)\right)\right| d s \\
& \leq k_{f}|x(t)-y(t)| \int_{0}^{t} g(t, s) d s \\
& +\widetilde{F} \int_{0}^{t} k_{v}(s)|x(s)-y(s)| d s+p_{a}\left(d\left(\lambda, \lambda_{1}\right)\right) \\
& +p_{f}\left(d\left(\lambda, \lambda_{1}\right)\right) \int_{0}^{t} g(t, s) d s+\widetilde{F} \int_{0}^{t} p_{v}\left(d\left(\lambda, \lambda_{1}\right)\right) d s \\
& \leq|x(t)-y(t)| k_{f} \sup _{t \in[0, T]} \int_{0}^{t} g(t, s) d s \\
& +\widetilde{F} \int_{0}^{t} k_{v}(s)|x(s)-y(s)| d s+p_{a}\left(d\left(\lambda, \lambda_{1}\right)\right) \\
& +\frac{p_{f}\left(d\left(\lambda, \lambda_{1}\right)\right)}{k_{f}}+\widetilde{F} T p_{v}\left(d\left(\lambda, \lambda_{1}\right)\right)
\end{aligned}
$$

where

$$
\widetilde{F}:=p_{f}(1)+\sup \{|f(\lambda, t, x)|: t \in[0, T], x \in \mathbb{R}\}<\infty .
$$


Summarizing, for fixed $\lambda \in \Lambda$ and $x \in \operatorname{Fix}(\lambda)$ and for arbitrary $\lambda_{1} \in B(\lambda, 1), y \in C([0, T])$, we derive

$$
\begin{aligned}
& \left|x(t)-\left(V_{\lambda_{1}} y\right)(t)\right| \\
& \quad \leq q|x(t)-y(t)|+\widetilde{F} \int_{0}^{t} k_{v}(s)|x(s)-y(s)| d s+b\left(\lambda_{1}\right),
\end{aligned}
$$

where we denoted

$$
\begin{gathered}
q:=k_{f} \sup _{t \in[0, T]} \int_{0}^{t} g(t, s) d s \\
b\left(\lambda_{1}\right):=p_{a}\left(d\left(\lambda, \lambda_{1}\right)\right)+\frac{p_{f}\left(d\left(\lambda, \lambda_{1}\right)\right)}{k_{f}}+\widetilde{F} T p_{v}\left(d\left(\lambda, \lambda_{1}\right)\right) .
\end{gathered}
$$

Consider the following equation:

$$
\psi_{\lambda_{1}}(t)=\frac{\widetilde{F}}{1-q} \int_{0}^{t} k_{v}(s) \psi_{\lambda_{1}}(s) d s+\frac{b\left(\lambda_{1}\right)}{1-q}, \quad t \in[0, T],
$$

where $\psi_{\lambda_{1}}:[0, T] \rightarrow \mathbb{R}_{+}$is unknown function. Standard calculations show that solution $\psi_{\lambda_{1}}$ of the above equation is given by formula

$$
\psi_{\lambda_{1}}(t)=\frac{b\left(\lambda_{1}\right)}{1-q} \exp \left(\frac{\widetilde{F}}{1-q} \int_{0}^{t} k_{v}(s) d s\right), \quad t \in[0, T] .
$$

Keeping in mind $\lim _{\lambda_{1} \rightarrow \lambda} b\left(\lambda_{1}\right)=0$ we infer that there exists sufficiently small number $\delta>0, \delta<1$ satisfying inequality

$$
\frac{b\left(\lambda_{1}\right)}{1-q} \exp \left(\frac{\tilde{F}}{1-q} \int_{0}^{T} k_{v}(s) d s\right)<\varepsilon, \quad \text { for } \lambda_{1} \in B(\lambda, \delta) .
$$

Now, let us fix $\lambda_{1} \in B(\lambda, \delta)$ and define the set

$$
\Omega:=\left\{y \in C([0, T]):|x(t)-y(t)| \leq \psi_{\lambda_{1}}(t), t \in[0, T]\right\} .
$$

We will prove

$$
V_{\lambda_{1}}(\Omega) \subset \Omega \text {. }
$$

Indeed, for $y \in \Omega$ and $t \in[0, T]$, in view of (58) and (60) we obtain

$$
\begin{aligned}
& \left|x(t)-\left(V_{\lambda_{1}} y\right)(t)\right| \\
& \quad \leq q|x(t)-y(t)|+\widetilde{F} \int_{0}^{t} k_{v}(s)|x(s)-y(s)| d s+b\left(\lambda_{1}\right) \\
& \quad \leq q \psi_{\lambda_{1}}(t)+\widetilde{F} \int_{0}^{t} k_{v}(s) \psi_{\lambda_{1}}(s) d s+b\left(\lambda_{1}\right)=\psi_{\lambda_{1}}(t)
\end{aligned}
$$

and it confirms (64).
If $x_{1}$ is a fixed point in $V_{\lambda_{1}}$ then, in view of (62), we obtain

$$
\left\|x-x_{1}\right\|=\sup _{t \in[0, T]}\left|x(t)-x_{1}(t)\right| \leq \sup _{t \in[0, T]} \psi_{\lambda_{1}}(t)<\varepsilon
$$

which confirms (54). The proof of estimate (55) is analogous.

Finally we will consider (40) on the real half-axis $\mathbb{R}_{+}$. First we express the necessary assumptions.

$\left(A_{1}^{\prime}\right) a: \Lambda \times \mathbb{R}_{+} \rightarrow \mathbb{R}$ is a function such that the function $t \rightarrow a(\lambda, t)$ is continuous on $\mathbb{R}_{+}$for arbitrarily fixed $\lambda \in \Lambda$ and moreover there exists a nondecreasing function $p_{a}: \mathbb{R}_{+} \rightarrow \mathbb{R}_{+}$, continuous at 0 and $p_{a}(0)=$ 0 , such that

$$
\left|a\left(\lambda_{1}, t\right)-a\left(\lambda_{2}, t\right)\right| \leq p_{a}\left(d\left(\lambda_{1}, \lambda_{2}\right)\right)
$$

for $\lambda_{1}, \lambda_{2} \in \Lambda, t \in \mathbb{R}_{+}$.

$\left(A_{2}^{\prime}\right) f: \Lambda \times \mathbb{R}_{+} \times \mathbb{R} \rightarrow \mathbb{R}$ is a function such that the function $(t, x) \rightarrow f(\lambda, t, x)$ is continuous and bounded on the set $\mathbb{R}_{+} \times \mathbb{R}$ for arbitrarily fixed $\lambda \epsilon$ $\Lambda$ and there are a constant $k_{f}$ and a nondecreasing function $p_{f}: \mathbb{R}_{+} \rightarrow \mathbb{R}_{+}$, continuous at 0 and $p_{f}(0)=$ 0 , such that

$$
|f(\lambda, t, x)-f(\lambda, t, y)| \leq k_{f}|x-y|
$$

for any $\lambda \in \Lambda, t \in \mathbb{R}_{+}, x, y \in \mathbb{R}$, and

$$
\left|f\left(\lambda_{1}, t, x\right)-f\left(\lambda_{2}, t, x\right)\right| \leq p_{f}\left(d\left(\lambda_{1}, \lambda_{2}\right)\right)
$$

for all $\lambda_{1}, \lambda_{2} \in \Lambda, t \in \mathbb{R}_{+}, x \in \mathbb{R}$.

$\left(A_{3}^{\prime}\right) v: \Lambda \times \mathbb{R}_{+} \times \mathbb{R}_{+} \times \mathbb{R} \rightarrow \mathbb{R}$ is a function such that the function $(t, s, x) \rightarrow \mathbb{R}$ is continuous on the set $\mathbb{R}_{+} \times \mathbb{R}_{+} \times \mathbb{R}$ for arbitrarily fixed $\lambda \in \Lambda$ and there are a continuous function $g: \mathbb{R}_{+} \times \mathbb{R}_{+} \rightarrow \mathbb{R}_{+}$and a nondecreasing function $p_{v}: \mathbb{R}_{+} \rightarrow \mathbb{R}_{+}$, continuous at 0 and $p_{v}(0)=0$, such that

$$
|v(\lambda, t, s, x)| \leq g(t, s)
$$

for all $\lambda \in \Lambda, t, s \in \mathbb{R}_{+}, x \in \mathbb{R}$ and

$$
\left|v\left(\lambda_{1}, t, s, x\right)-v\left(\lambda_{2}, t, s, x\right)\right| \leq p_{v}\left(d\left(\lambda_{1}, \lambda_{2}\right)\right)
$$

for all $\lambda_{1}, \lambda_{2} \in \Lambda, t, s \in \mathbb{R}_{+}, x \in \mathbb{R}$.

$\left(A_{4}^{\prime}\right)$ Consider

$$
k_{f} \sup _{t \in[0, T]} \int_{0}^{t} g(t, s) d s<1, \quad T \in \mathbb{R}_{+} .
$$


$\left(A_{5}^{\prime}\right)$ There is an integrable function $k_{v}: \mathbb{R}_{+} \rightarrow \mathbb{R}_{+}$such that

$$
|v(\lambda, t, s, x)-v(\lambda, t, s, y)| \leq k_{v}(s)|x-y|
$$

for $\lambda \in \Lambda, t, s \in \mathbb{R}_{+}, x, y \in \mathbb{R}$.

Under the above assumptions we can formulate a theorem analogous to the previous one.

Theorem 11. Under assumptions $\left(A_{1}^{\prime}\right)-\left(A_{5}^{\prime}\right),(40)$ has at least one solution in the space $C\left(\mathbb{R}_{+}\right)$for all parameters $\lambda \in \Lambda$ and, moreover, the set of all solutions $\operatorname{Fix}(\lambda)$ depends continuously (with respect to Hausdorff metric $D_{C\left(\mathbb{R}_{+}\right)}$) on the parameter $\lambda$.

Proof. The proof of this theorem uses Lemma 6 and it can be done in the same way as the proof of Theorem 10. We omit details.

Remark 12. Notice that, in hypotheses $\left(A_{1}\right)-\left(A_{3}\right)$ in Theorem 10, we have assumed uniform continuity of the functions $a, f$, and $v$ with respect to variable $\lambda$. Therefore, there appears a natural question.

Question. Is Theorem 10 true in the case when we assume only continuity of the functions $a, f$, and $v$ instead of uniform continuity?

Finally, we provide an example of an integral equation of the form (40) for which the assumptions of Theorem 10 are satisfied.

Example 13. Consider the following functional integral equation:

$$
\begin{array}{r}
x(t)=\lambda t^{2}+\lambda \sin \left(\frac{t x(t)}{3}\right) \int_{0}^{t} \sqrt[3]{s e^{-t}+\frac{\lambda(1+|x(s)|)}{2+|x(s)|}} d s, \\
t \in[0,1],
\end{array}
$$

where $\lambda \in \Lambda:=[1,2]$. The space $\Lambda$ is equipped with the standard Euclidean metric. Observe that (74) is a special case of (40), where $a(\lambda, t)=\lambda t^{2}, f(\lambda, t, x)=\lambda \sin (t x / 3)$, and $v(\lambda, t, s, x)=\sqrt[3]{s e^{-t}+\lambda(1+|x|) /(2+|x|)}$. Moreover

$$
\begin{array}{r}
\left|a\left(\lambda_{1}, t\right)-a\left(\lambda_{2}, t\right)\right| \leq p_{a}\left(\left|\lambda_{1}-\lambda_{2}\right|\right), \\
\text { where } p_{a}(\tau)=\tau, \\
\left|f\left(\lambda_{1}, t, x\right)-f\left(\lambda_{2}, t, x\right)\right| \leq p_{f}\left(\left|\lambda_{1}-\lambda_{2}\right|\right), \\
\text { where } p_{f}(\tau)=\tau, \\
\left|v\left(\lambda_{1}, t, s, x\right)-f\left(\lambda_{2}, t, s, x\right)\right| \leq p_{v}\left(\left|\lambda_{1}-\lambda_{2}\right|\right), \\
\text { where } p_{f}(\tau)=\frac{\sqrt[3]{2} \tau}{3},
\end{array}
$$

$$
\begin{aligned}
& |f(\lambda, t, x)-f(\lambda, t, y)| \leq \frac{2}{3}|x-y|, \quad \text { i.e. } k_{f}=\frac{2}{3}, \\
& |v(\lambda, t, s, x)|=\sqrt[3]{s e^{-t}+\frac{\lambda(1+|x|)}{2+|x|}} \leq \sqrt[3]{3}, \\
& \text { i.e., } g(t, s)=\sqrt[3]{3}, \\
& |v(\lambda, t, s, x)-v(\lambda, t, s, y)| \\
& \quad \leq \frac{|\lambda(1+|x|) /(2+|x|)-\lambda(1+|y|) /(2+|y|)|}{\sup _{t \in[1]} \int_{0}^{t} g(t, s) d s=\sqrt[3]{3}<\frac{1}{k_{f}}=\frac{3}{2},} \\
& \leq \frac{\sqrt[3]{e^{2}}}{6} s^{-2 / 3}|x-y| ;
\end{aligned}
$$

that is, the function $k_{v}(s)=\left(\sqrt[3]{e^{2}} / 6\right) s^{-2 / 3}$ for $s \in(0,1]$ is integrable.

Joining all above facts we infer that assumptions $\left(A_{1}\right)-\left(A_{5}\right)$ of Theorem 10 are satisfied. Finally, applying Theorem 10 we conclude that the set of all solutions $\operatorname{Fix}(\lambda)$ of (74) depends continuously on the parameter $\lambda$.

\section{Conflict of Interests}

The authors declare that there is no conflict of interests regarding the publication of this paper.

\section{References}

[1] R. P. Agarwal, D. O'Regan, and P. J. Y. Wong, Positive Solutions of Differential, Difference and Integral Equations, Kluwer Academic, Dordrecht, The Netherlands, 1999.

[2] K. Deimling, Nonlinear Functional Analysis, Springer, Berlin , Germany, 1985.

[3] M. Väth, Volterra and Integral Equations of Vector Functions, Pure and Applied Mathematics, Marcel Decker, New York, NY, USA, 2000.

[4] P. P. Zabrejko, A. I. Koshelev, M. A. Krasnoselskii, S. G. Mikhlin, L. S. Rakovschik, and V. J. Stetsenko, Integral Equations, Nordhoff, Leyden, Holland, 1975.

[5] J. Banaś and S. Dudek, "The technique of measures of noncompactness in Banach algebras and its applications to integral equations," Abstract and Applied Analysis, vol. 2013, Article ID 537897, 15 pages, 2013.

[6] J. Banaś, J. Rocha Martin, and K. Sadarangani, "On solutions of a quadratic integral equation of Hammerstein type," Mathematical and Computer Modelling, vol. 43, no. 1-2, pp. 97-104, 2006.

[7] J. Banaś and B. Rzepka, "Monotonic solutions of a quadratic integral equation of fractional order," Journal of Mathematical Analysis and Applications, vol. 332, no. 2, pp. 1371-1379, 2007.

[8] B. C. Dhage and V. Lakshmikantham, "On global existence and attractivity results for nonlinear functional integral equations," Nonlinear Analysis: Theory, Methods \& Applications, vol. 72, no. 5, pp. 2219-2227, 2010. 
[9] W. G. El-Sayed, "Solvability of a neutral differential equation with deviated argument," Journal of Mathematical Analysis and Applications, vol. 327, no. 1, pp. 342-350, 2007.

[10] J. Banaś and K. Goebel, Measure of Noncompactness in Banach Spaces, vol. 60 of Lecture Notes in Pure and Applied Mathematics, Marcel Dekker, New York, NY, USA, 1980.

[11] X. Hu and J. Yan, "The global attractivity and asymptotic stability of solution of a nonlinear integral equation," Journal of Mathematical Analysis and Applications, vol. 321, no. 1, pp. 147156, 2006.

[12] L. Olszowy, "Fixed point theorems in the Fréchet space $C\left(R_{+}\right)$ and functional integral equations on an unbounded interval," Applied Mathematics and Computation, vol. 218, no. 18, pp. 9066-9074, 2012.

[13] L. Olszowy, "On solutions of functional-integral equations of Urysohn type on an unbounded interval," Mathematical and Computer Modelling, vol. 47, no. 11-12, pp. 1125-1133, 2008. 


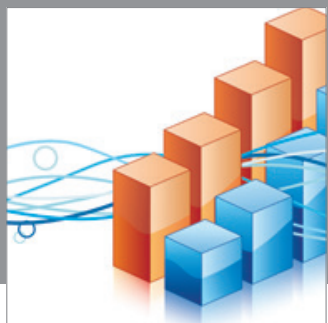

Advances in

Operations Research

mansans

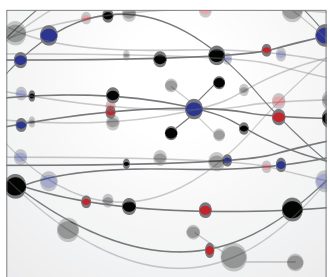

The Scientific World Journal
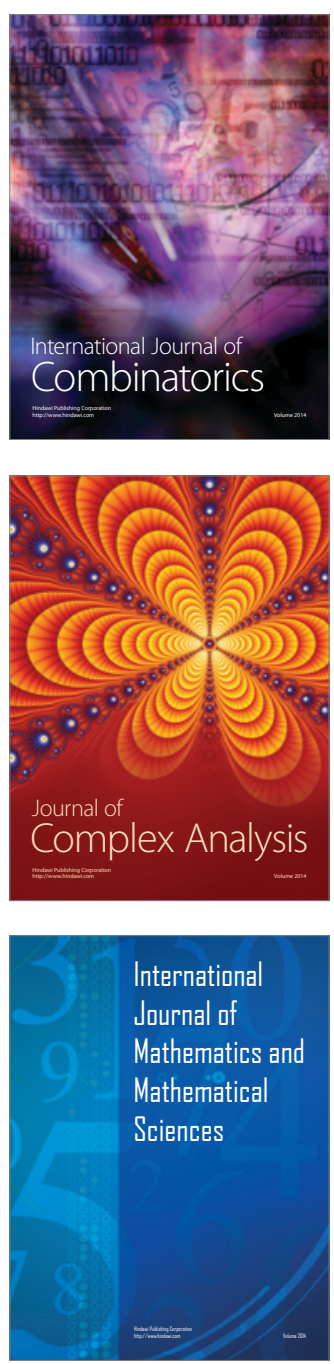
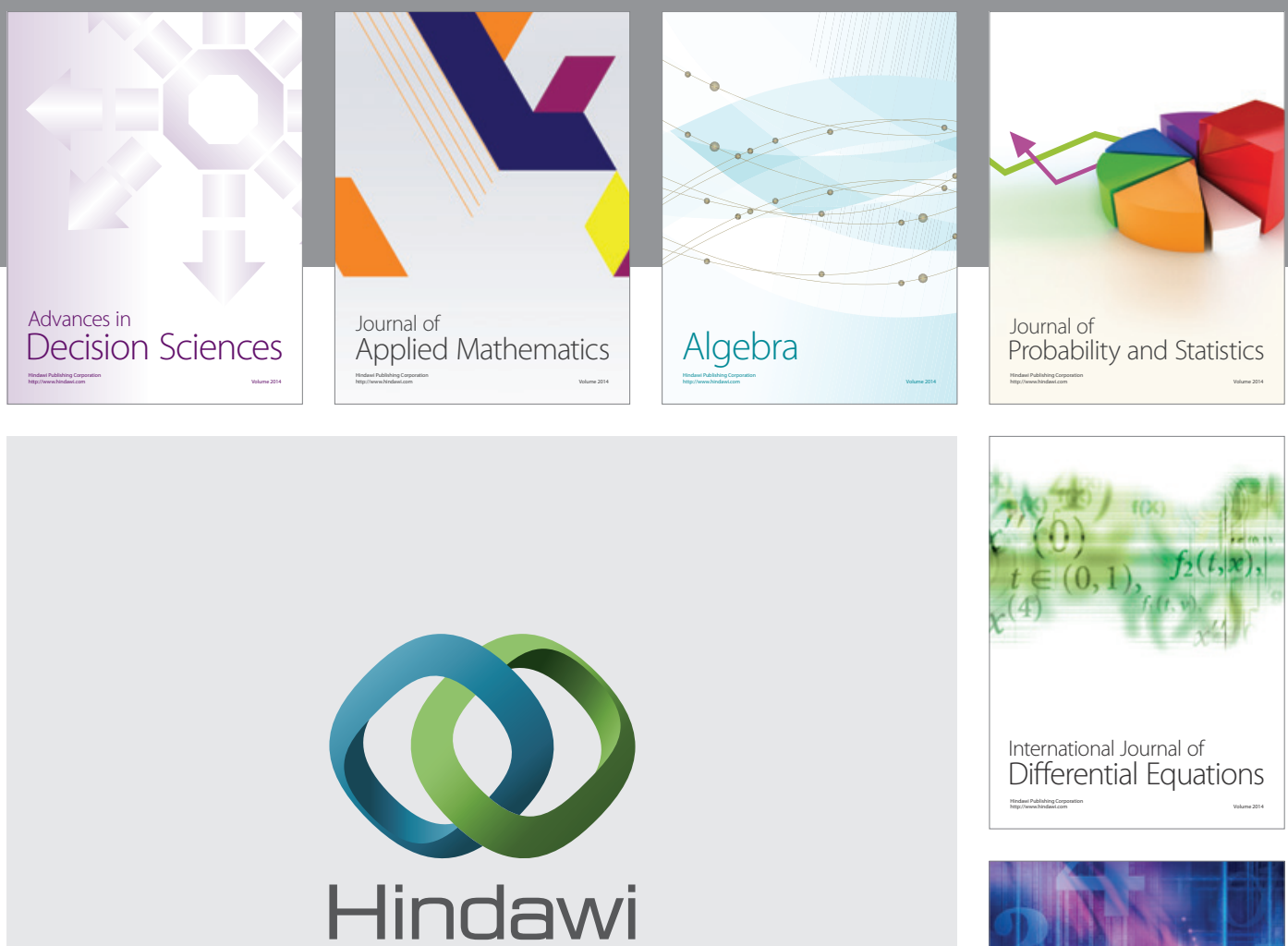

Submit your manuscripts at http://www.hindawi.com
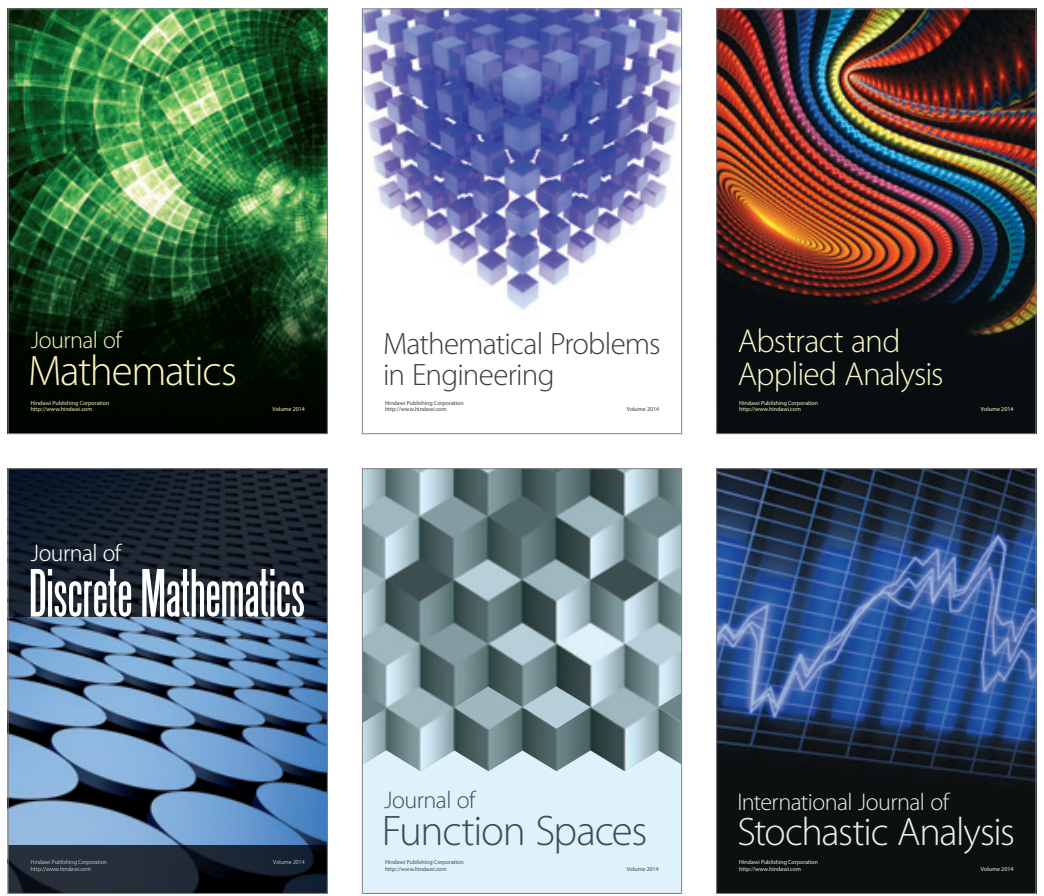

Journal of

Function Spaces

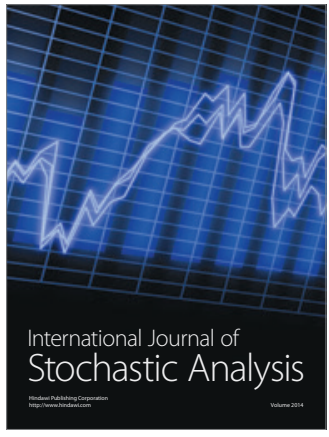

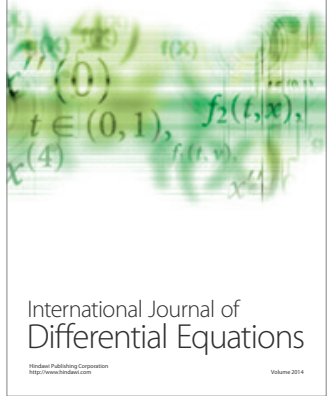
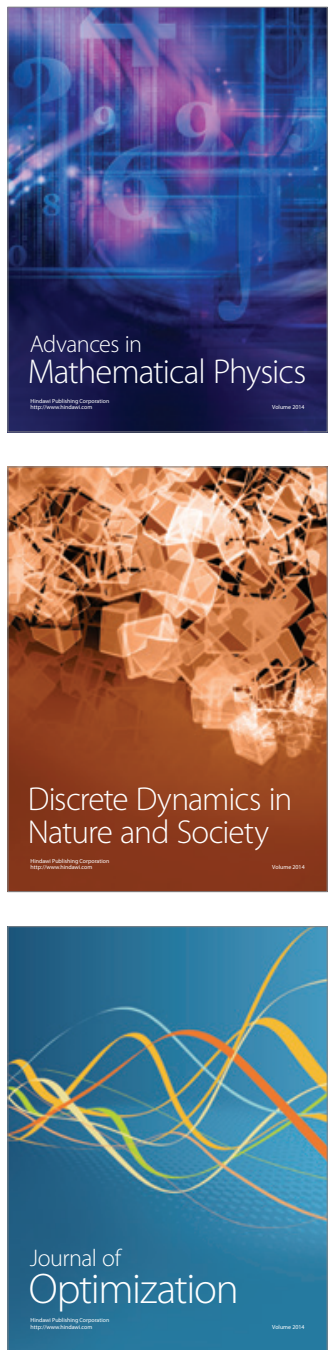